\title{
Investigating the causes of neurodevelopmental deficits in congenital heart disease through multiple gestations
}

\author{
Ryan R. Davies, MD
}

\footnotetext{
From Nemours Cardiac Center, A.I. duPont Hospital for Children, Wilmington, Del; and Thomas Jefferson University, Philadelphia, Pa.

Disclosures: Author has nothing to disclose with regard to commercial support.

Received for publication March 2, 2017; accepted for publication March 10, 2017; available ahead of print May $16,2017$.

Address for reprints: Ryan R. Davies, MD, Nemours Cardiac Center, A.I. duPont Hospital for Children, 1600 Rockland Rd, Wilmington, DE 19803 (E-mail: rdavies@nemours.org).

J Thorac Cardiovasc Surg 2017;154:282-3

$0022-5223 / \$ 36.00$

Copyright $(2) 2017$ by The American Association for Thoracic Surgery

http://dx.doi.org/10.1016/j.jtcvs.2017.03.026
}

With improved survival among children undergoing infantile cardiac surgery for congenital heart disease (CHD), the importance of long-term neurodevelopmental (ND) deficits has become increasingly clear. Unfortunately, recognition has not led to clarity regarding the cause of these deficits. They are likely multifactorial, and covariation between many risk factors makes isolating particular ones challenging. With this background, the study by Dr Schultz and colleagues ${ }^{1}$ in this issue of the Journal represents an important and novel attempt to control for some of these factors by evaluating children who are products of multiple gestation births in which 1 child has CHD. By comparing children requiring infantile cardiopulmonary bypass with their same gestation siblings, the methodology controls for maternal gestational environment, parental intelligence, education and socioeconomic status, and the home environment. The study confirms that independently of these differences, children requiring cardiac surgery with cardiopulmonary bypass early in life have long-term ND deficits apparent at 4 years of age.

There are limitations to the methodology, most of which are well described by the authors, including (1) the inability to control for differences in the precise in utero environment-especially in the absence of knowledge regarding amniosity and zygosity; (2) the lack of genetic data resulting in the possibility of unidentified genetic causes of both CHD and intellectual disability in one versus the other twin; (3) the inability to control for differences in how caregivers may have interacted with the "sick" versus "healthy" sibling, which may result in dissimilar early developmental environments even in twins; and (4) the small sample size, which may limit the statistical significance of clinically significant differences between the siblings (auditory/visual impairment) or between the analyzed multiple gestations and singletons with CHD (ethnicity, socioeconomic status). Therefore, the study should not be taken to suggest that the environment (whether in utero or postoperative) does not with CHD.

\section{References}

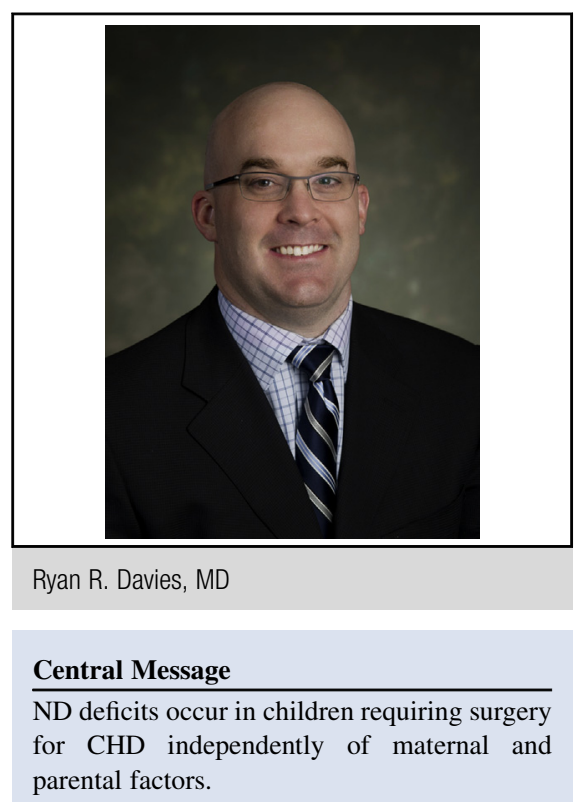

See Article page 273

affect ND outcomes in children with CHD. Rather, it should be read conservatively as demonstrating that maternal age and gestational environment, prematurity, and parental factors do not explain the entirety of the ND deficits in children

The study contains another result of potentially greater impact. The siblings with CHD had median scores on the ND evaluations that were at or around the normative baseline in nearly all domains. This occurred despite the high incidence of prematurity, which is known to be associated with worse ND outcomes, ${ }^{2}$ as well as significant deficits at 1-year evaluations in an overlapping cohort. $^{3}$ This suggests that normative ND outcomes are achievable 4 years after infantile cardiac surgery. Differences between the multiple gestation cohort and the wider cohort suggest potential positive factors, including higher socioeconomic status and educational achievement. Future studies with similarly innovative methodology will be required to continue to elucidate the causes of ND deficits and understand how to mitigate them. The current study by Schultz et al $^{1}$ is an important step on that pathway.

1. Schultz AH, Ittenbach RF, Gerdes M, Jarvik GP, Wernovsky G, Bernbaum J, et al Effect of congenital heart disease on 4-year neurodevelopment within multiplegestation births. J Thorac Cardiovasc Surg. 2017;154:273-81.e2. 
2. Goff DA, Luan X, Gerdes M, Bernbaum J, D’Agostino JA, Rychik J, et al. Younger gestational age is associated with worse neurodevelopmental outcomes after cardiac surgery in infancy. J Thorac Cardiovasc Surg. 2012;143:535-42.
3. Schultz AH, Jarvik GP, Wernovsky G, Bernbaum J, Clancy RR, D’Agostino JA, et al. Effect of congenital heart disease on neurodevelopmental outcomes within multiple-gestation births. J Thorac Cardiovasc Surg. 2005;130:1511-6. 